\title{
Nanoencapsulation of Luminescent 3-Hydroxypicolinate Lanthanide Complexes
}

\author{
Kingsley O. Iwu, ${ }^{\dagger}$ Paula C. R. Soares-Santos, ${ }^{\dagger}$ Helena I. S. Nogueira, ${ }^{\dagger}$ Luís D. Carlos, ${ }^{*,+}$ and \\ Tito Trindade $*, \dagger$ \\ Department of Chemistry and CICECO, University of Aveiro, 3810-193 Aveiro, Portugal, and Department of \\ Physics and CICECO, University of Aveiro, 3810-193 Aveiro, Portugal
}

Received: December 23, 2008; Revised Manuscript Received: March 4, 2009

\begin{abstract}
We have synthesized luminescent nanoparticles comprising a core of lanthanide complexes and shells of amorphous silica using reverse micelles as nanoreactors. 3-Hydroxypicolinate complexes of Eu(III), Tb(III), and the corresponding heteronuclear complexes have been investigated as the photoactive cores. The size of the silica particles is within the nanometer scale, which, together with the ability for surface biofunctionalization, opens up perspectives for their use in bioapplications. Optical studies of the as-prepared nanoparticles reveal that the luminescence properties of the 3-hydroxypicolinate complexes in the matrices are markedly different from their original features.
\end{abstract}

\section{Introduction}

The synergy between nanotechnology and the biological sciences is especially important in pushing the frontiers in medicine, ${ }^{1-3}$ environment ${ }^{4}$ and food technology. ${ }^{5}$ For biomedical sciences, especially, nanotechnology holds a lot of potential in areas such as drug delivery, gene delivery, and bioassays., ${ }^{2,3,6,7}$ One obvious advantage of employing nanoparticles in bioassays is that nanoparticles are within dimensions of biomolecules and cellular components and, thus, can act as local probes and sensors. ${ }^{3,8}$ This is especially true for homogeneous assays where, in addition to their small size, nanoparticles also provide large surface areas (by volume of material) for interaction with specific biomolecules.

Water-in-oil microemulsions (W/O's) or reverse micelles have become very important and widely used in the preparation of nanoparticles. Monodispersed particles can be prepared when syntheses are carried out in the nanosized water pools that can be regarded as dynamic nanoreactors. ${ }^{6,9-13}$ They also offer the possibility of size tuning by varying the [water]/[surfactant] ratio $R$; the lower the $R$ value, the smaller the average size of the nanoparticles. ${ }^{6,14}$ In this context, W/O's have been used in the preparation of silica, ${ }^{6,15}$ titania,,${ }^{11}$ perovskites, ${ }^{16}$ semiconductor nanoparticles, ${ }^{10,17,18}$ and even organic nanoparticles. ${ }^{1}$ Consequently, $\mathrm{SiO}_{2}$ nanoparticles prepared in W/O's have been reported to encapsulate a wide variety of luminescent compounds, ranging from semiconductor nanocrystals, ${ }^{19,20} \mathrm{YF}_{3}$ nanoparticles, ${ }^{9}$ organic/inorganic dyes, and lanthanide (Ln) chelates. ${ }^{21,22}$

In this work, we have investigated this approach to encapsulate lanthanide chelates in silica nanoparticles prepared by the Stober method. 3-Hydroxypicolinate complexes of europium(III) and terbium(III), as well as the mixed-lanthanide $(\mathrm{Eu}$ and $\mathrm{Tb})$ complexes, were used for silica encapsulation. The discussion on 3-hydroxypicolinate complexes will be largely skewed in favor of $\mathrm{Eu}^{3+}$ due to the high luminescent behavior of both the pure complex and the derived nanocomposites.

* Corresponding authors. Phone: +351 234370726 (T.T.), +351 234 370946 (L.D.C.). Fax: +351 234370084 (T.T.), +351 234378197

(L.D.C.). E-mail: tito@ua.pt (T.T.), 1carlos@ua.pt (L.D.C.).

$\uparrow$ Department of Chemistry and CICECO.

* Department of Physics and CICECO.
Considering that, in general, lanthanide complexes show high luminescence quantum yields, narrow bandwidth and long-lived emission, large Stokes' shifts, and ligand-dependent luminescence sensitization, ${ }^{23,24}$ these nanodispersed systems can be of interest as new bioprobes.

\section{Experimental Section}

2.1. Materials. 3-Hydroxypicolinic acid $(\mathrm{HpicOH}$, Aldrich, 98\%), sodium bis(2-ethylhexyl) sulfosuccinate (AOT, Aldrich, 96\%), europium(III) chloride hexahydrate (Aldrich, 99.9\%), terbium(III) chloride hexahydrate (Aldrich, 99.9\%), and tetraethyl orthosilicate (TEOS, Aldrich, 98\%) were obtained from commercial sources and used without further purification.

2.2. Synthesis of the Lanthanide Complexes. The 3-hydroxypicolinate complexes, $\left[\mathrm{Ln}\left(\mathrm{H}_{2} \mathrm{O}\right)(\mathrm{picOH})_{2}(\mu-\mathrm{HpicO})\right] \cdot n \mathrm{H}_{2} \mathrm{O}$ $(\mathrm{Ln}=\mathrm{Eu}(\mathbf{1}), \mathrm{Tb}(\mathbf{2}))$, were prepared as reported previously. ${ }^{25}$ A similar procedure was employed for the synthesis of the mixed-lanthanide complexes, $\mathrm{Eu}_{0.5} \mathrm{~Tb}_{0.5}(3), \mathrm{Eu}_{0.3} \mathrm{~Tb}_{0.7}(\mathbf{4})$, and $\mathrm{Eu}_{0.1} \mathrm{~Tb}_{0.9}$ (5), by adding the stoichiometric amounts of $\mathrm{EuCl}_{3} \cdot 6 \mathrm{H}_{2} \mathrm{O}$ and $\mathrm{TbCl}_{3} \cdot 6 \mathrm{H}_{2} \mathrm{O}$. The lanthanide hydroxides used as starting compounds were prepared by adding aqueous solutions $(8 \mathrm{~mL})$ of $\mathrm{KOH}(4.5 \mathrm{mmol})$ to an equal volume of aqueous solutions containing the lanthanide chloride salt (1.5 $\mathrm{mmol}$ ). The solid formed was stirred for over $90 \mathrm{~min}$, filtered, and washed thoroughly with distilled water. The freshly prepared $\mathrm{Ln}(\mathrm{OH})_{3}(1 \mathrm{mmol})$ was added to an aqueous solution $(25 \mathrm{~mL})$ containing 3-hydroxypicolinic acid ( $4 \mathrm{mmol})$. This mixture was stirred during $1 \mathrm{~h}$, then heated for $30 \mathrm{~min}$ at $80^{\circ} \mathrm{C}$, and further stirred over $8 \mathrm{~h}$ at room temperature. The obtained solid was filtered, washed thoroughly with distilled water, and dried over silica gel.

2.3. Preparation of Silica Nanoparticles. Water-in-oil microemulsions were prepared by dissolving $0.6 \mathrm{~g}$ of AOT in 10 $\mathrm{mL}$ of heptane, followed by addition of distilled water $(50,100$, 150,200 , and $245 \mu \mathrm{L}$, corresponding to $R=2,4,6,8$, and 10 , respectively) under vigorous stirring. Next, $5 \mathrm{mg}$ of the lanthanide complex or $10 \mathrm{mg}$ of a mixture of the europium complex and the terbium complex [5:5 (6), 3:7 (7), and 1:9 (8)] dissolved in dimethyl sulfoxide (DMSO) was added, followed by addition of $100 \mu \mathrm{L}$ of TEOS and, finally, $100 \mu \mathrm{L}$ of a $\mathrm{NH}_{4} \mathrm{OH}$ aqueous solution (25\%). Each step had a 5-7 min 
interval between them. After the mixture was stirred over $24 \mathrm{~h}$, the silica nanoparticles were precipitated out by addition of acetone and then separated by centrifugation. The luminescent silica nanoparticles $\left(1 / \mathrm{SiO}_{2}-R\right)$ were washed twice with heptane and ethanol, followed by 1-propanol, 1-butanol, and ethanol.

2.4. Instrumentation. FT-IR spectra were obtained as $\mathrm{KBr}$ pellets using a Mattson 7000 FT instrument. Raman spectra were recorded using a Bruker RFS100/S FT-Raman spectrometer (Nd: YAG laser, $1064 \mathrm{~nm}$ excitation). Elemental analyses for $\mathrm{C}, \mathrm{H}$, and $\mathrm{N}$ were performed with a CHNS-932 elemental analyzer in the Microanalysis Laboratory of the University of Aveiro. The lanthanide content for the heteronuclear complexes was obtained by ICP (inductively coupled plasma) analysis on an ICP-AES Jobin Yvon model $70+$ at the Central Analytical Laboratory of the University of Aveiro.

The scanning electron microscopy (SEM) images were obtained on a Hitachi SU 70 microscope fitted with an energydispersive spectroscopy (EDX) accessory (EDX Detector, Bruker AXS, Quantax software). The transmission electron microscopy (TEM) study of the nanoparticles' morphology was carried out with a Hitachi H-9000-NA TEM or using a HR(EF) TEM JOEL 2200FS, both operating at $200 \mathrm{kV}$. Samples were prepared by dipping carbon-coated copper grids in dilute ethanol solutions of the samples and then letting the solvent evaporate. Average particle sizes were determined by statistical calculations based on the TEM images only.

The excitation and emission spectra were obtained at $300 \mathrm{~K}$ on a Jobin Yvon-Spex spectrometer (HR 460) coupled to a R928 Hamamatsu photomultiplier under continuous excitation of a $\mathrm{Xe}$ arc lamp $(150 \mathrm{~W})$. The excitation monochromator was a Jobin Yvon (TRIAX 180). The spectra were corrected for the response of the detector. The lifetime measurements and the excitation and emission spectra of $\mathbf{2}$ were recorded at $300 \mathrm{~K}$ on a modular double-grating excitation spectrofluorimeter with a TRIAX 320 emission monochromator (Fluorolog-3, Jobin YvonSpex) coupled to a R928 Hamamatsu photomultiplier using the front face acquisition mode. All the photoluminescence spectra were collected on solid samples at room temperature and corrected for optical and detection spectral responses. The roomtemperature lifetime measurements were acquired with the setup described for the luminescence spectra using a pulsed $\mathrm{Xe}-\mathrm{Hg}$ lamp (6 $\mu$ s pulse at half-width and a $20-30 \mu$ s tail). The ${ }^{5} \mathrm{D}_{0}\left(\mathrm{Eu}^{3+}\right)$ lifetimes were monitored within the more intense emission line of the ${ }^{5} \mathrm{D}_{0} \rightarrow{ }^{7} \mathrm{~F}_{2}$ transition, under the excitation that maximizes the $\mathrm{Eu}^{3+}$ emission. The emission decay curves (not shown) were well-reproduced by single exponentials.

\section{Results and Discussion}

3.1. Synthesis and Material Characterization. A series of $\mathrm{Eu}(\mathrm{III})$ and $\mathrm{Tb}$ (III) complexes with 3-hydroxypicolinic acid were synthesized and structurally characterized by elemental analysis and infrared, Raman, and photoluminescence spectroscopy. Subsequently, the lanthanide complexes, $\left[\mathrm{Ln}\left(\mathrm{H}_{2} \mathrm{O}\right)(\mathrm{picOH})_{2}(\mu\right.$ $\mathrm{HpicO})] \cdot n \mathrm{H}_{2} \mathrm{O}(\mathbf{1}-\mathbf{5})$, were encapsulated into amorphous silica nanoparticles (complex $/ \mathrm{SiO}_{2}-R$, where $R=2,4,6,8$, and 10), as schematically illustrated in Figure 1.

Table 1 shows the infrared and Raman spectroscopic data for 3-hydroxypicolinic acid and its lanthanide complexes together with the elemental analysis for $\mathrm{C}, \mathrm{H}, \mathrm{N}$, and $\mathrm{Ln}$. Elemental analysis results are in accordance with the molecular formula $\left[\mathrm{Ln}\left(\mathrm{H}_{2} \mathrm{O}\right)(\mathrm{picOH})_{2}(\mu-\mathrm{HpicO})\right] \cdot n \mathrm{H}_{2} \mathrm{O}$ for all the lanthanide complexes $(\mathbf{1 - 5})$, which is consistent with the vibrational data presented below. Additionally, the vibrational data for the mixed-lanthanide complexes $(\mathbf{3}-\mathbf{5})$ are in good agree-

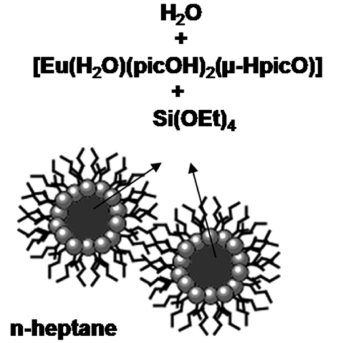

Figure 1. Schematic representation of reverse micelles as nanoreactors for the $\mathrm{SiO}_{2}$ encapsulation of $\mathrm{Ln}$ complexes.

ment with those described for the pure lanthanide $(\mathrm{Eu}$ and $\mathrm{Tb})$ complexes already reported in the literature. ${ }^{25}$

To confirm the chemical homogeneity of the heteronuclear bulk samples, the spatial distribution of europium and terbium in the mixed-lanthanide complexes $\mathbf{3 - 5}$, and in the derived nanoparticles, was analyzed by EDX. Figure 2 shows the SEM image and corresponding EDX mapping for the Eu and $\mathrm{Tb}$ elements of a typical nanosilica sample $\left(\mathbf{5} / \mathrm{SiO}_{2}-10\right)$. These results show that both lanthanide ions are homogeneously distributed in the final $\mathrm{SiO}_{2}$ nanoparticles, as expected, in a material containing those ions homogeneously mixed at the molecular level. For comparison, the EDX mapping for the Eu and $\mathrm{Tb}$ elements in the starting complexes $(\mathbf{3}-\mathbf{5})$ used for the encapsulation is shown in the Supporting Information (Figures S1-S3).

TEM analysis revealed particles with a spherical morphology, as illustrated in Figure 3, for the sample $\mathbf{3} / \mathrm{SiO}_{2}-10$. Additionally, these TEM results also confirmed the core-shell structure of these hybrid lanthanide/ $\mathrm{SiO}_{2}$ materials that comprise a nanometric core assigned to the encapsulated Ln complex. The $R$ value was used to change the particle size, and spheroidal core/ shell type nanoparticles were observed for the several samples analyzed. Because of the high sensitivity of the morphology of these nanostructures to the synthetic conditions employed, the particle size of the nanoparticles could vary considerably. It was observed that the diameter of the composite particles could range from 30 to $55 \mathrm{~nm}$, with an average size of $45 \mathrm{~nm}$ and a particle size dispersion superior to $10 \%$. Nevertheless, the TEM results (Figure 3a) showed core-shell structures with a typical diameter of about $9 \mathrm{~nm}$ for the core of the composite particles. For the sake of comparison, similar syntheses made in the absence of the complex result in $\mathrm{SiO}_{2}$ particles whose TEM images (Figure $3 b)$ did not show the darker cores. On the other hand, samples of the pure complex did not appear as morphologically uniform particles, but still, we cannot exclude the presence of nonencapsulated complexes dispersed in these samples. In fact, the results presented here provide the basis for the $\mathrm{SiO}_{2}$ nanoencapsulation of lanthanide complexes using reverse micelles, but we hope to continue our investigations to optimize the nanocomposites' morphological characteristics. Because the surfactant molecules can compete for the lanthanide sites, it will be interesting, for example, to see how the morphological homogeneity is favored by varying the chemical nature of the lanthanide complex and/or the surfactant.

3.2. Photoluminescence. Photoluminescence measurements were carried out for the silica nanoparticles with different sizes encapsulating the europium(III) 3-hydroxypicolinate complex $\left(1 / \mathrm{SiO}_{2}-R ; R=2,4,6\right.$, and 10$)$ and compared with those of the pure europium complex. The excitation and emission spectra of $1 / \mathrm{SiO}_{2}-R$ together with $\mathbf{1}$ are shown in Figure 4 . The excitation spectra of the materials (Figure 4A) show a large broad band ranging from 250 to $400 \mathrm{~nm}$ and a series of sharp 

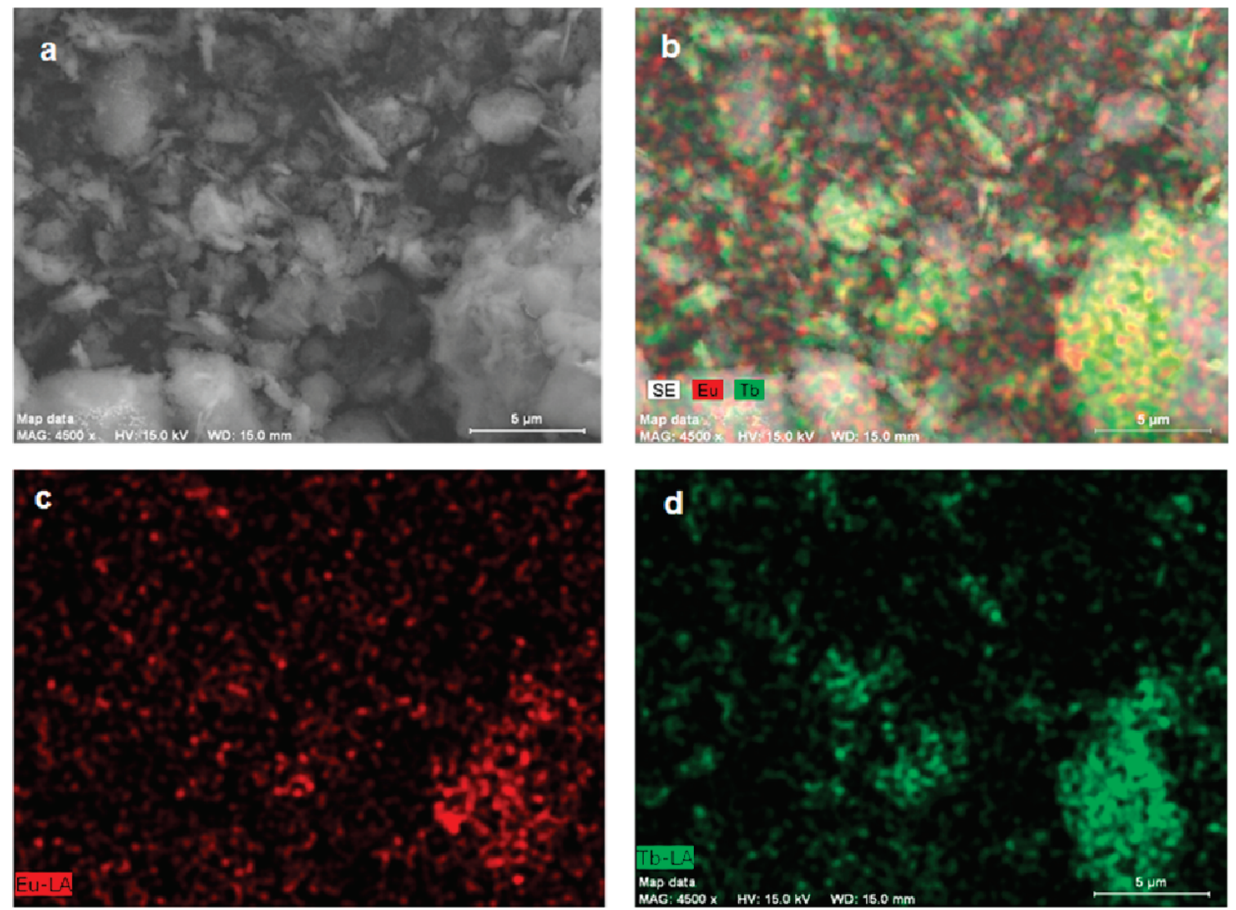

Figure 2. (a) SEM image and corresponding EDX distribution maps of (b) Eu: $\mathrm{Tb}$, (c) Eu, and (d) $\mathrm{Tb}$ in $\mathbf{5} / \mathrm{SiO}_{2}-10$.

TABLE 1: Analytical and Vibrational Data for the Free Ligand and Its Lanthanide Complexes (1-5)

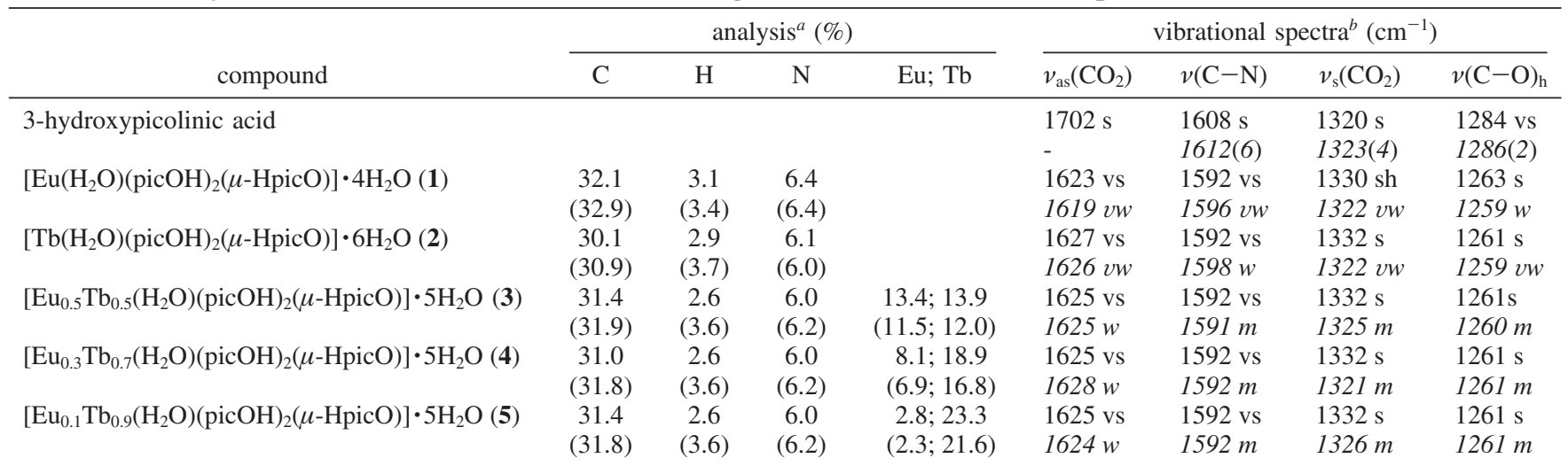

${ }^{a}$ Calculated values in parentheses. ${ }^{b}$ Infrared and Raman (in italics) data: vs, very strong; s, strong; m, medium; w, weak; sh, shoulder.

lines assigned to intra- $4 \mathrm{f}^{6}$ transitions. The large broad band can be ascribed to the excited levels of the ligand $(\mathrm{L}),{ }^{25}$ indicating the existence of ligand-to-Eu ${ }^{3+}$ energy transfer. The higher intensity of the large broad band relative to that of the intra- $4 \mathrm{f}^{6}$ transitions reveals that the luminescence sensitization via the ligands' excited states is more efficient than $\mathrm{f}-\mathrm{f}$ direct excitation. The peak position corresponding to the maximum intensity of the broad band ranges from 363 to $380 \mathrm{~nm}$ for the $1 / \mathrm{SiO}_{2}-R$ series ( $375 \mathrm{~nm}$ for 1). Excitations within the ligands' absorption band $(325-380 \mathrm{~nm})$ for $\mathbf{1} / \mathrm{SiO}_{2}$ and $\mathbf{1}$ lead to the typical $\mathrm{Eu}^{3+}$ emission spectra $\left({ }^{5} \mathrm{D}_{0} \rightarrow{ }^{7} \mathrm{~F}_{0-4}\right)$, without any broad emission band arising from the organic ligands (Figure 4B). No significant changes are detected on the emission spectra of the $1 / \mathrm{SiO}_{2}-R$ series exciting within the ligands' absorption band or within the direct europium absorption lines (not shown). However, differences are detected in the relative intensity of the ${ }^{5} \mathrm{D}_{0} \rightarrow$ ${ }^{7} \mathrm{~F}_{2,4}$ transitions for the $1 / \mathrm{SiO}_{2}$ series by varying the size of the nanoparticles. The small differences detected in the emission and excitation spectra of the $1 / \mathrm{SiO}_{2}-R$ series indicate that the local environment of the $\mathrm{Eu}^{3+}$ is similar even though it is not the same. Further studies will be performed to better understand these differences that lie outside the scope of this work.
A meticulous analysis of the emission spectra of the nanoparticles shows some variations when compared with that of the starting europium complex, namely, of the energy $(E)$ and full width at half-maximum (fwhm) of the ${ }^{5} \mathrm{D}_{0} \rightarrow{ }^{7} \mathrm{~F}_{0}$ transition and maximum splitting of the ${ }^{5} \mathrm{D}_{0} \rightarrow{ }^{7} \mathrm{~F}_{1}$ transition $(\Delta E)$. The corresponding values are listed in Table 2 , where $1 / \mathrm{SiO}_{2}-10$ was selected to represent the $1 / \mathrm{SiO}_{2}$ series. The fwhm of the ${ }^{5} \mathrm{D}_{0} \rightarrow$ ${ }^{7} \mathrm{~F}_{0}$ transition for $\mathbf{1}$ is $20 \mathrm{~cm}^{-1}$, whereas for $\mathbf{1} / \mathrm{SiO}_{2}-10$, it is 30 $\mathrm{cm}^{-1}$, revealing an increased nonhomogeneity for the $\mathrm{Eu}^{3+}$ local coordination site. ${ }^{26}$ This fact brings to the fore the question of whether the process is a mere encapsulation, as opposed to a sort of chemical reaction between the matrix and the europium complex leading to extensive modification of the local environment of $\mathrm{Eu}^{3+}$. Changes in the ${ }^{5} \mathrm{D}_{0} \rightarrow{ }^{7} \mathrm{~F}_{0}$ energy are highly dependent on the covalency of the $\mathrm{Eu}^{3+}-\mathrm{L}$ bonds of the first $\mathrm{Eu}^{3+}$ coordination sphere. A red shift of the ${ }^{5} \mathrm{D}_{0} \rightarrow{ }^{7} \mathrm{~F}_{0}$ energy with respect to the energy of the gaseous $\mathrm{Eu}^{3+}\left(17374 \mathrm{~cm}^{-1}\right)$ means an increase in covalency. ${ }^{27-29}$ Thus, the blue shift found for the ${ }^{5} \mathrm{D}_{0} \rightarrow{ }^{7} \mathrm{~F}_{0}$ transition in $\mathbf{1} / \mathrm{SiO}_{2}$ relative to the europium pure complex is an indication of a decreasing covalency of the $\mathrm{Eu}^{3+}-\mathrm{L}$ bonds in the first $\mathrm{Eu}^{3+}$ coordination sphere in the silica matrix. A similar shift was found for the silica nanocomposite 


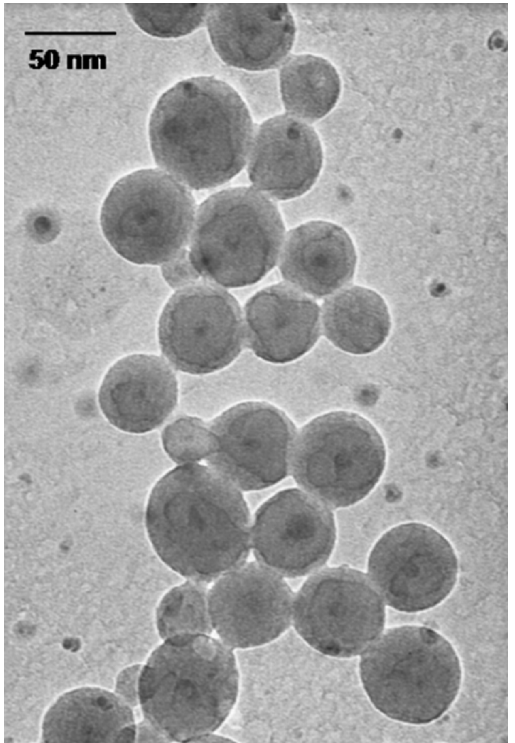

(a)

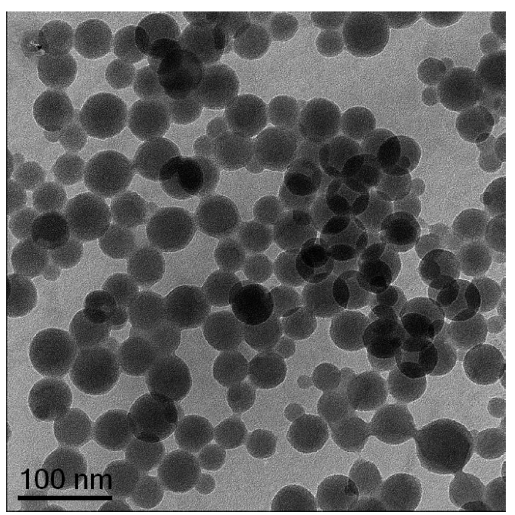

(b)

Figure 3. TEM images of $\mathbf{3} / \mathrm{SiO}_{2}-10$ (a) and $\mathrm{SiO}_{2}$ particles (b).

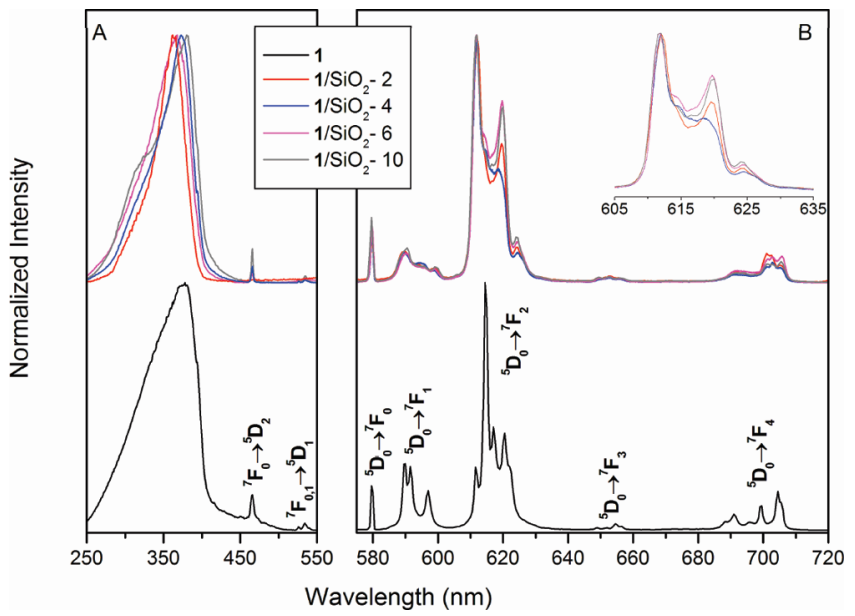

Figure 4. (A) Excitation and (B) emission spectra of $\mathbf{1}$ and $\mathbf{1} / \mathrm{SiO}_{2}-R$ monitored at 614.5 (for $\mathbf{1}$ ) and $612 \mathrm{~nm}$ (for $\mathbf{1} / \mathrm{SiO}_{2}-R$ ) and excited at $375(1), 363(R=2), 373(R=4), 368(R=6)$, and $380 \mathrm{~nm}(R=10)$. The inset in B shows a detailed view of the ${ }^{5} \mathrm{D}_{0} \rightarrow{ }^{7} \mathrm{~F}_{2}$ transition.

containing the europium complex in our earlier work, ${ }^{25}$ where it was suggested that the europium complex in the nanocomposite is bound to the silica surface via the silanol groups,

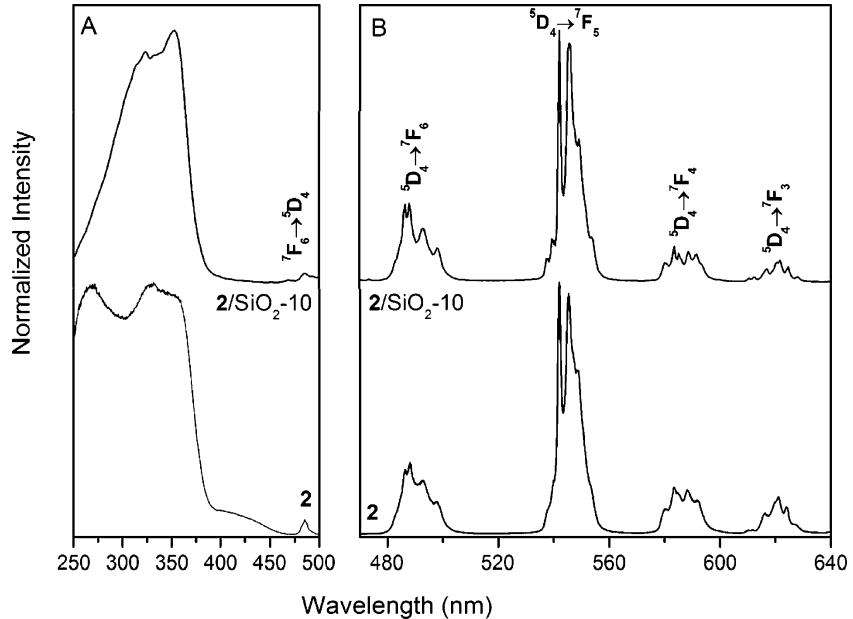

Figure 5. (A) Excitation and (B) emission spectra of $\mathbf{2}$ and $\mathbf{2} / \mathrm{SiO}_{2}-10$ monitored at $544 \mathrm{~nm}$ and excited at $320 \mathrm{~nm}$, respectively.

TABLE 2: Energy $(E)$ and fwhm of the ${ }^{5} D_{0} \rightarrow{ }^{7} F_{0}$ Transition and Maximum Splitting $(\Delta E)$ of the ${ }^{5} \mathrm{D}_{0} \rightarrow{ }^{7} \mathrm{~F}_{1}$ Transition for Selected Materials

\begin{tabular}{lccc}
\hline & \multicolumn{2}{c}{${ }^{5} \mathrm{D}_{0} \rightarrow{ }^{7} \mathrm{~F}_{0}$} & ${ }^{5} \mathrm{D}_{0} \rightarrow{ }^{7} \mathrm{~F}_{1}$ \\
\cline { 2 - 3 } sample & $E\left(\mathrm{~cm}^{-1}\right)$ & fwhm $\left(\mathrm{cm}^{-1}\right)$ & $\Delta E\left(\mathrm{~cm}^{-1}\right)$ \\
\hline $\mathbf{1}$ & 17249 & 20 & 205 \\
$\mathbf{1} / \mathrm{SiO}_{2}-10$ & 17258 & 30 & 260 \\
$\mathbf{5}$ & 17258 & 21 & 325 \\
$\mathbf{5} / \mathrm{SiO}_{2}-10$ & 17247 & 34 & 236 \\
$\mathbf{8} / \mathrm{SiO}_{2}-10$ & 17258 & 22 & 347
\end{tabular}

possibly displacing one bridging carboxylate group from the $\mathrm{Eu}^{3+}$ first coordination sphere. Therefore, similar interactions should occur between the europium complex and the silica nanoparticles $\left(\mathbf{1} / \mathrm{SiO}_{2}-R\right)$. The maximum splitting of the ${ }^{5} \mathrm{D}_{0} \rightarrow$ ${ }^{7} \mathrm{~F}_{1}$ transition has been reported as being directly proportional to the strength of the ligand (crystal) field interaction. ${ }^{26,29}$ This value increased for the $1 / \mathrm{SiO}_{2}$ series relative to the pure europium complex, indicating a greater crystal field interaction strength than that in $\mathbf{1}$. The main ${ }^{5} \mathrm{D}_{0} \rightarrow{ }^{7} \mathrm{~F}_{2}$ emission peak shifts from $16273 \mathrm{~cm}^{-1}(\sim 614.5 \mathrm{~nm})$ for 1 to about $16340 \mathrm{~cm}^{-1}$ $(\sim 612 \mathrm{~nm})$ in the $\mathbf{1} / \mathrm{SiO}_{2}$ series. Despite all the spectroscopic evidence indicating that the $\mathrm{Eu}^{3+}$ local coordination changes from 1 to $1 / \mathrm{SiO}_{2}$, the local field splitting of the ${ }^{5} \mathrm{D}_{0} \rightarrow{ }^{7} \mathrm{~F}_{1}$ and ${ }^{5} \mathrm{D}_{0} \rightarrow{ }^{7} \mathrm{~F}_{2}$ transitions into three and five Stark components, respectively, points out that the $\mathrm{Eu}^{3+}$ ions occupy, in both materials, a low site symmetry without an inversion center (high intensity of the ${ }^{5} \mathrm{D}_{0} \rightarrow{ }^{7} \mathrm{~F}_{2}$ transition ${ }^{25}$ ).

The experimental lifetime of the excited ${ }^{5} \mathrm{D}_{0}\left(\mathrm{Eu}^{3+}\right)$ energy level, $\tau_{\text {exp }}$, for $1 / \mathrm{SiO}_{2}-10$ is $0.450 \pm 0.003 \mathrm{~ms}$, slightly longer than the corresponding value measured for $1(0.431 \pm 0.002$ $\mathrm{ms})$. The quantum efficiency, $q$, of the excited ${ }^{5} \mathrm{D}_{0}$ state, $q=$ $k_{\mathrm{r}} /\left(k_{\mathrm{r}}+k_{\mathrm{nr}}\right)$, where $k_{\mathrm{r}}$ and $k_{\mathrm{nr}}$ are the radiative and nonradiative transition probabilities, respectively, was calculated on the basis of the emission spectrum and ${ }^{5} \mathrm{D}_{0}$ lifetime $\left(\tau_{\mathrm{exp}}^{-1}=A_{\mathrm{r}}+A_{\mathrm{nr}}\right){ }^{25,26,29}$ The $k_{\mathrm{r}}$ contribution is calculated from the relative intensities of the ${ }^{5} \mathrm{D}_{0} \rightarrow{ }^{7} \mathrm{~F}_{0-4}$ transitions (the ${ }^{5} \mathrm{D}_{0} \rightarrow{ }^{7} \mathrm{~F}_{5,6}$ branching ratios are neglected due to their poor relative intensity with respect to that of the remaining ${ }^{5} \mathrm{D}_{0} \rightarrow{ }^{7} \mathrm{~F}_{0-4}$ lines). The ${ }^{5} \mathrm{D}_{0} \rightarrow{ }^{7} \mathrm{~F}_{1}$ transition does not depend on the local ligand field and, thus, may be used as a reference for the whole spectrum. An effective refractive index of 1.5 was used, leading to $A_{01} \approx 50 \mathrm{~s}^{-1} ; A_{01}$ stands for the Einstein's coefficient of spontaneous emission between the ${ }^{5} \mathrm{D}_{0}$ and the ${ }^{7} \mathrm{~F}_{1}$ Stark levels.

We found an increase in the calculated ${ }^{5} \mathrm{D}_{0}$ quantum efficiency for $\mathbf{1} / \mathrm{SiO}_{2}-10(23 \%)$ when compared with that of $\mathbf{1}(11 \%)$ due 


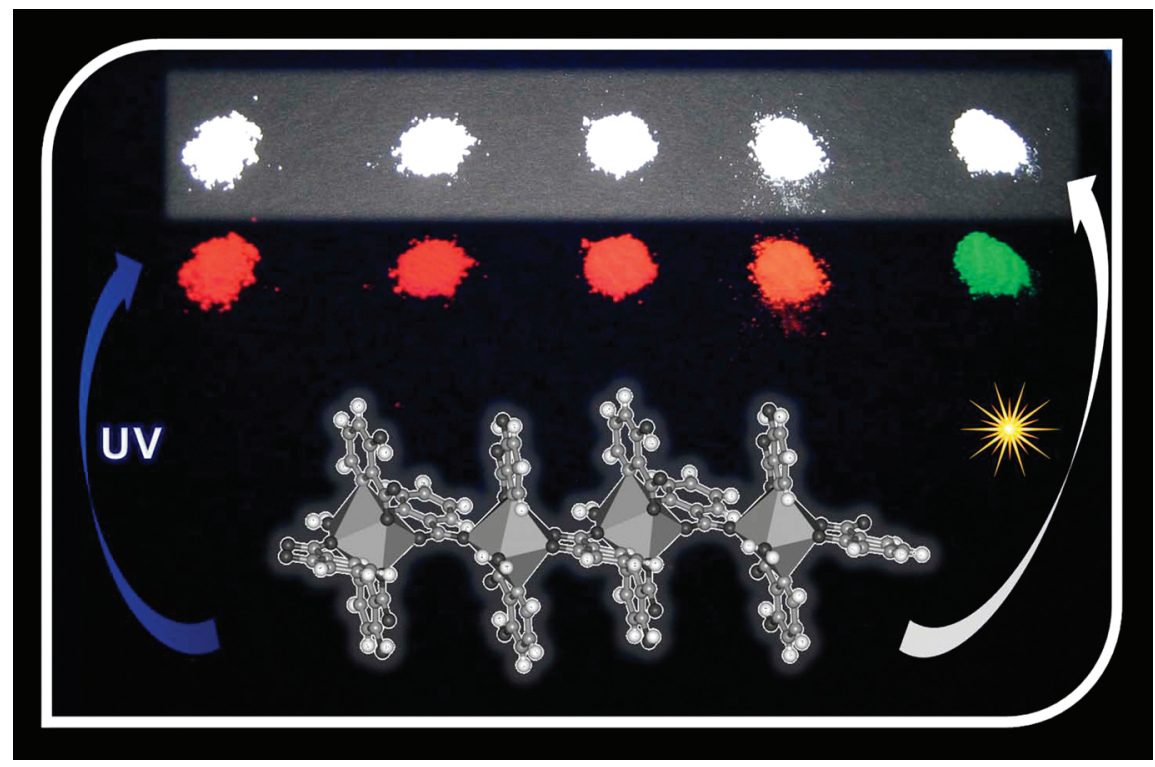

Figure 6. Powdered lanthanide complexes under UV light and natural light, following the sequence 1, 3, 4, 5, and 2, from left to right.

to an increase for the former material of the radiative transition probability, $k_{\mathrm{r}}$, and to a decrease of the nonradiative one, $k_{\mathrm{nr}}$ $\left(0.518\right.$ and $1.70 \mathrm{~ms}^{-1}$, respectively, for $\mathbf{1} / \mathrm{SiO}_{2}-10$ and 0.497 and $2.07 \mathrm{~ms}^{-1}$ for $\mathbf{1}$ ).

The increase in $q$ and $\tau_{\text {exp }}$ for the europium complex when it is embedded in a silica matrix (via a sol-gel approach) over those of a pure europium complex corroborates earlier findings reported by us. ${ }^{25}$

The terbium(III) complex (2) was also incorporated into the silica matrix $(R=2,4,6,8$, and 10). The excitation and emission spectra of $\mathbf{2}$ and $2 / \mathrm{SiO}_{2}-10$ are shown in Figure 5. The excitation spectra of the nanoparticles (Figure 5A) show a large broad band ranging from 250 to $380 \mathrm{~nm}$ (already ascribed to the excited levels of the ligands) and the ${ }^{7} \mathrm{~F}_{6} \rightarrow{ }^{5} \mathrm{D}_{4}$ transition with low intensity relative to the large broad band. This large broad band, also observed in $\mathbf{2}$ and in the 1-based materials described above, shows similar profiles for $R=6,8$, and 10 (Figure S4A, Supporting Information) and is double-crested with the first peak around $320 \mathrm{~nm}$ and the second around $350 \mathrm{~nm}$. Indeed, the excitation spectra profile of $1 / \mathrm{SiO}_{2}-10$ may be considered as approximately a single-peak (maximum at 380 $\mathrm{nm}$ ) with a shoulder around $320 \mathrm{~nm}$ (Figure 4A). Figure 5B shows the emission spectra of $\mathbf{2}$ and $2 / \mathrm{SiO}_{2}-10$ excited at 320 $\mathrm{nm}$, which display the typical intra- $4 \mathrm{f}^{8}$ lines of the $\mathrm{Tb}^{3+}$ ion $\left({ }^{5} \mathrm{D}_{4} \rightarrow{ }^{7} \mathrm{~F}_{6-3}\right)$. Varying the particle size or the excitation wavelength does not produce significant changes in the emission spectra of the terbium-based nanoparticles (Figure S4B, Supporting Information).

The $\mathrm{Eu}^{3+} / \mathrm{Tb}^{3+}$ mixed complexes $3\left(\mathrm{Eu}_{0.5} \mathrm{~Tb}_{0.5}\right), \mathbf{4}\left(\mathrm{Eu}_{0.3} \mathrm{~Tb}_{0.7}\right)$, and $5\left(\mathrm{Eu}_{0.1} \mathrm{~Tb}_{0.9}\right)$ (prepared and characterized for the first time in this paper) were encapsulated into silica nanoparticles $(R=$ 8 and 10). Figure 6 exhibits the digital photographs of the powdered lanthanide complexes 3-5 under UV light and natural light. Figure 7A displays the excitation spectra of $\mathbf{3}, \mathbf{4}$, and $\mathbf{5}$ monitoring the ${ }^{5} \mathrm{D}_{0} \rightarrow{ }^{7} \mathrm{~F}_{2}(620 \mathrm{~nm})$ transition. The observed large broad band is mainly formed of a peak at $357 \mathrm{~nm}$ and a shoulder around $330 \mathrm{~nm}$. The existence of the $\mathrm{Tb}^{3+}$-to- $\mathrm{Eu}^{3+}$ energy transfer is supported by the detection of the $\mathrm{Tb}^{3+}{ }^{7} \mathrm{~F}_{6} \rightarrow$ ${ }^{5} \mathrm{D}_{4}$ transition located at $486 \mathrm{~nm}$ on the excitation spectra of $\mathbf{3}$, $\mathbf{4}$, and $\mathbf{5}$ (when the $\mathrm{Eu}^{3+}$ emission is being monitored).

The emission spectra of all the mixed-lanthanide complexes excited within the ligands' broad band (357 nm) (Figure 7B)

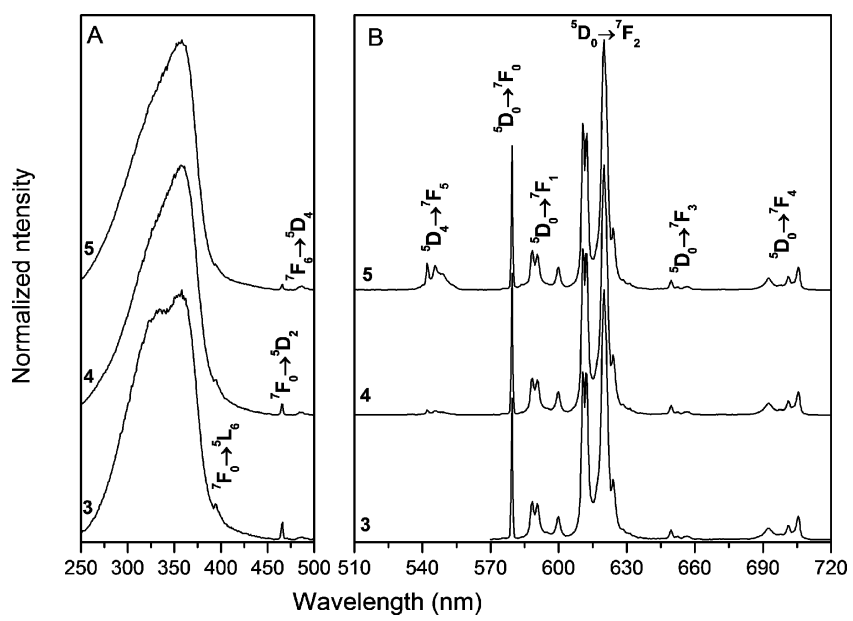

Figure 7. (A) Excitation and (B) emission of 3, 4, and $\mathbf{5}$ monitored at $620 \mathrm{~nm}$ and excited at $357 \mathrm{~nm}$, respectively.

exhibit, besides the $\mathrm{Tb}^{3+}{ }^{5} \mathrm{D}_{4} \rightarrow{ }^{7} \mathrm{~F}_{5}$ lines, the typical $\mathrm{Eu}^{3+}{ }^{5} \mathrm{D}_{0}$ $\rightarrow{ }^{7} \mathrm{~F}_{0-4}$ transitions, suggesting the occurrence of ligand-to- $\mathrm{Tb}^{3+}$, ligand-to- $\mathrm{Eu}^{3+}$, and $\mathrm{Tb}^{3+}$-to- $\mathrm{Eu}^{3+}$ energy transfer. The most intense emission line, which is the main Stark component of the ${ }^{5} \mathrm{D}_{0} \rightarrow{ }^{7} \mathrm{~F}_{2}$ transition, is located at $16129 \mathrm{~cm}^{-1}(\sim 620 \mathrm{~nm})$, in contrast to $16273 \mathrm{~cm}^{-1}(\sim 614.5 \mathrm{~nm})$ for $\mathbf{1}$. Additionally, the energy of the ${ }^{5} \mathrm{D}_{0} \rightarrow{ }^{7} \mathrm{~F}_{0}$ transition for the mixed-lanthanide complexes increased above that of $\mathbf{1}$ (Table 2), although the fwhm was maintained. The $\Delta E$ of the ${ }^{5} \mathrm{D}_{0} \rightarrow{ }^{7} \mathrm{~F}_{1}$ transition for $5\left(325 \mathrm{~cm}^{-1}\right)$ increased well above the value for $\mathbf{1}\left(205 \mathrm{~cm}^{-1}\right)$, showing a stronger ligand field interaction in the mixedlanthanide complexes relative to that of $\mathbf{1}$. Therefore, it can be seen from the foregoing that the presence of the $\mathrm{Tb}^{3+}$ ions in the mixed-lanthanide complexes affects the local coordination of the $\mathrm{Eu}^{3+}$ ions and, hence, their optical features. It is noteworthy that the relative intensity of the ${ }^{7} \mathrm{~F}_{0} \rightarrow{ }^{5} \mathrm{D}_{2}$ transition decreases (Figure 7A) and that of the ${ }^{5} \mathrm{D}_{4} \rightarrow{ }^{7} \mathrm{~F}_{6}$ transition increases (Figure 7B) when the $\mathrm{Eu}^{3+}$ ions are gradually substituted by the $\mathrm{Tb}^{3+}$ ions.

All the mixed-lanthanide complexes were encapsulated into silica nanoparticles $(R=8$ and 10). Their excitation spectra (not shown) roughly approximate those of the mixed-lanthanide complexes whose peaks range from 355 to $370 \mathrm{~nm}$, and their 


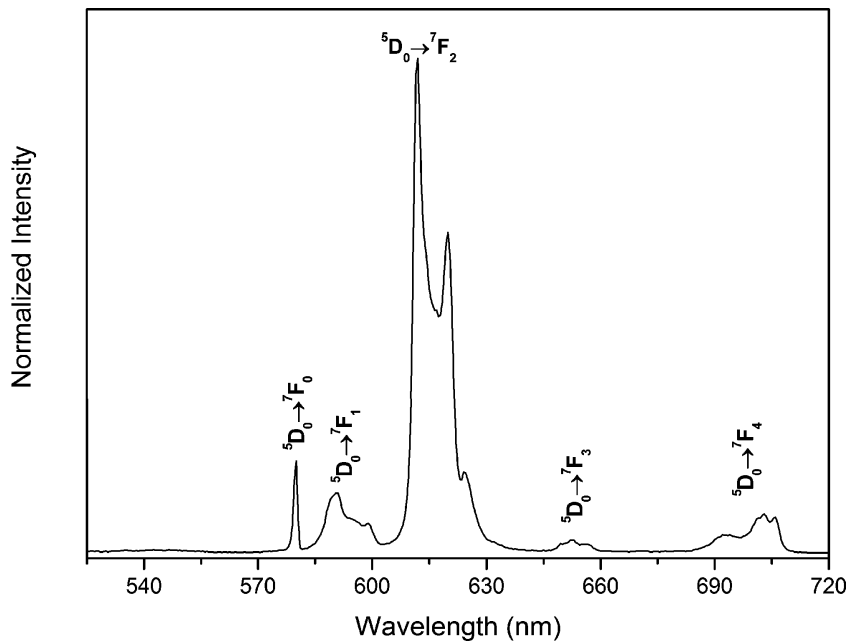

Figure 8. Emission spectra of $\mathbf{5} / \mathrm{SiO}_{2}-10$ excited at $357 \mathrm{~nm}$.

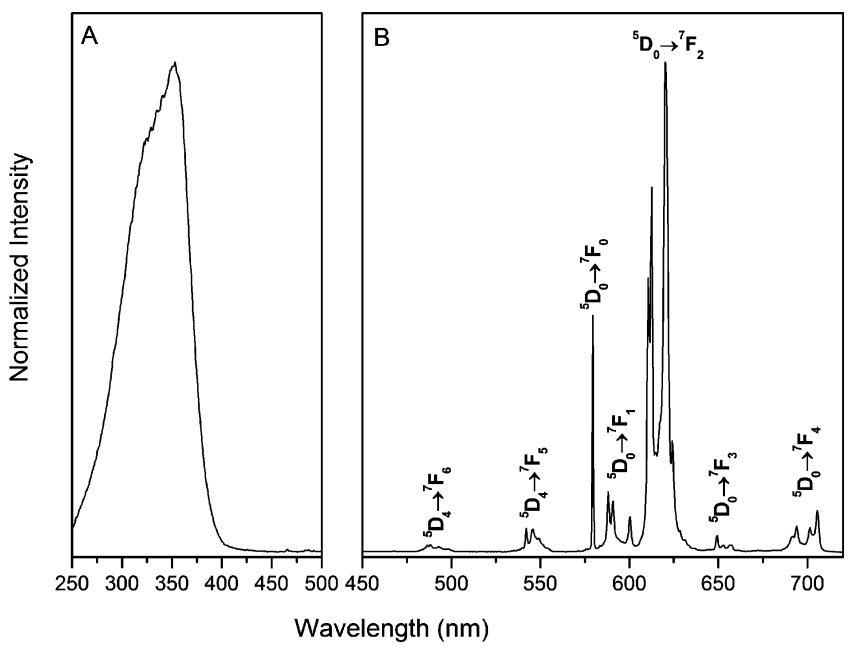

Figure 9. (A) Excitation and (B) emission spectra of $\mathbf{8} / \mathrm{SiO}_{2}-10$ monitored at $620 \mathrm{~nm}$ and excited at $353 \mathrm{~nm}$, respectively.

emission spectra (Figure S5 in the Supporting Information) are very similar. Nevertheless, changes are observed in the emission spectra of $\mathbf{5} / \mathrm{SiO}_{2}-10$ (Figure 8) relative to the isolated complex 5. When $\mathbf{5}$ is incorporated into the silica matrix, the fwhm of the ${ }^{5} \mathrm{D}_{0} \rightarrow{ }^{7} \mathrm{~F}_{0}$ transition increased up to $13 \mathrm{~cm}^{-1}$ (Table 2), revealing an increased nonhomogeneity for the $\mathrm{Eu}^{3+}$ local coordination site, whereas the energy of the ${ }^{5} \mathrm{D}_{0} \rightarrow{ }^{7} \mathrm{~F}_{0}$ transition decreased up to $11 \mathrm{~cm}^{-1}$, revealing that, on average, the $\mathrm{Eu}^{3+}-\mathrm{L}$ bonds in the nanocomposites experience some increase in covalency. The $\Delta E$ of the ${ }^{5} \mathrm{D}_{0} \rightarrow{ }^{7} \mathrm{~F}_{1}$ transition for $\mathbf{5} / \mathrm{SiO}_{2}-10$ decreased significantly to $236 \mathrm{~cm}^{-1}$ compared with that of $\mathbf{5}$ $\left(325 \mathrm{~cm}^{-1}\right)$; this indicates a lower crystal field interaction strength. The most intense emission line shifts from $16129 \mathrm{~cm}^{-1}$ $(\sim 620 \mathrm{~nm})$ for the mixed-lanthanide complexes to around $16340 \mathrm{~cm}^{-1}(612 \mathrm{~nm})$ for the mixed-lanthanide silica nanoparticles, and the number of Stark components detected for the ${ }^{5} \mathrm{D}_{0} \rightarrow{ }^{7} \mathrm{~F}_{1,2}$ transitions is one less than the number in the mixedlanthanide complexes. It is of considerable importance to emphasize that the $\mathrm{Tb}^{3+}{ }^{5} \mathrm{D}_{4} \rightarrow{ }^{7} \mathrm{~F}_{5}$ transition detected in the mixed-lanthanide complexes (Figure 7A) disappeared in the related nanocomposites (Figure S5 in the Supporting Information). For that reason, it can be assumed that the $\mathrm{Tb}^{3+}$-to- $\mathrm{Eu}^{3+}$ energy transfer in the $\mathbf{3}-\mathbf{5} / \mathrm{SiO}_{2}-R$ series is more efficient than that in the starting complexes and/or that nonradiative deactivation of the $\mathrm{Tb}^{3+}$ excited levels occurs.
The physical mixtures of the europium complex (1) and the terbium complex (2) in the stoichiometric amounts of $5: 5,3: 7$, and $1: 9$ were incorporated into the silica matrix, giving the materials named here as $6 / \mathrm{SiO}_{2}-R, 7 / \mathrm{SiO}-R$, and $8 / \mathrm{SiO}_{2}-R(R=6,8$, and 10$)$, respectively. The excitation and emission spectra of $\mathbf{8} / \mathrm{SiO}_{2}-10$ (Figure 9) are very similar to those of $6 / \mathrm{SiO}_{2}-10$ and $7 / \mathrm{SiO}_{2}-10$ (Figure $\mathrm{S} 6$ in the Supporting Information). The excitation spectrum of $\mathbf{8} / \mathrm{SiO}_{2}$ 10 monitoring the ${ }^{5} \mathrm{D}_{0} \rightarrow{ }^{7} \mathrm{~F}_{2}\left(\mathrm{Eu}^{3+}, 620 \mathrm{~nm}\right)$ transition only shows a large broad band mainly formed of a peak around $350 \mathrm{~nm}$ and a shoulder around $320 \mathrm{~nm}$. For $\mathbf{6} / \mathrm{SiO}_{2}-10$ and 7/SiO -10 (Figure $\mathrm{S} 6$ in the Supporting Information), the ${ }^{5} \mathrm{~L}_{6}$ and ${ }^{5} \mathrm{D}_{2} \mathrm{Eu}^{3+}$ levels and the ${ }^{5} \mathrm{D}_{4} \mathrm{~Tb}^{3+}$ level are detected, the latter being clear evidence of the occurrence of $\mathrm{Tb}^{3+}$-to- $\mathrm{Eu}^{3+}$ energy transfer. The profile of the large broad band splits into two crests, one centered around $322 \mathrm{~nm}$ and the other around $356 \mathrm{~nm}$; this is particularly seen for $6 / \mathrm{SiO}_{2}-6,6 / \mathrm{SiO}_{2^{-}}$ 8 , and $7 / \mathrm{SiO}_{2}-8$ (not shown). The similarity between the emission spectra of $\mathbf{8} / \mathrm{SiO}_{2}-10$ and $\mathbf{5}$ (Figures 9 and 7 and Table 2) is clear, particularly, for the values of $E$ and the fwhm of the ${ }^{5} \mathrm{D}_{0} \rightarrow{ }^{7} \mathrm{~F}_{0}$ transition. When we consider that the $\mathrm{Eu}^{3+} / \mathrm{Tb}^{3+}$ stoichiometric amounts in $\mathbf{8} / \mathrm{SiO}_{2}-10$ and $\mathbf{5}$ are retained, the decrease of the relative intensity of the ${ }^{5} \mathrm{D}_{4} \rightarrow$ ${ }^{7} \mathrm{~F}_{5}$ transition (when compared with that of the ${ }^{5} \mathrm{D}_{0} \rightarrow{ }^{7} \mathrm{~F}_{2}$ transition) of 0.05 in $\mathbf{8} / \mathrm{SiO}_{2}-10$ (compared with the value of 0.11 for 5 ) shows that the $\mathrm{Tb}^{3+}$-to- $\mathrm{Eu}^{3+}$ energy transfer in $\mathbf{8} / \mathrm{SiO}_{2}-10$ is more efficient than that in $\mathbf{5}$ and/or that nonradiative deactivation of the $\mathrm{Tb}^{3+}$ excited levels occurs. In contrast to that observed for the $\mathbf{3}-\mathbf{5} / \mathrm{SiO}_{2}$ series, the detection of the $\mathrm{Tb}^{3+}{ }^{5} \mathrm{D}_{4} \rightarrow{ }^{7} \mathrm{~F}_{6,5}$ transitions points out the (i) lower efficiency of the $\mathrm{Tb}^{3+}$-to-Eu ${ }^{3+}$ energy transfer, (ii) occurrence of radiative deactivation of the $\mathrm{Tb}^{3+}$ excited levels, or (iii) existence of clustering of a given $\mathrm{Ln}^{3+}$ in a separate material domain. In conclusion, the $\mathrm{Eu}^{3+}$ local environment in this series suffered less alteration than in the remaining nanoparticles, as can be seen from the values of the fwhm of the ${ }^{5} \mathrm{D}_{0} \rightarrow{ }^{7} \mathrm{~F}_{0}$ transition, which are significantly lower than those of the $\mathbf{1} / \mathrm{SiO}_{2}$ and $\mathbf{5} / \mathrm{SiO}_{2}$ and closer to 1 and 5 .

\section{Conclusions}

Reverse microemulsions were used to encapsulate 3-hydroxypicolinate complexes of $\mathrm{Eu}(\mathrm{III})$ and/or $\mathrm{Tb}$ (III) complexes in silica nanoparticles. Our earlier work to prepare silica nanoparticles by encapsulating 1 using the sol-gel method $^{25}$ led to particles with an average diameter above 100 $\mathrm{nm}$; here, the preparation of particles with an average diameter of $45 \mathrm{~nm}$ and below confirmed that reverse micelles are useful for producing such nanoparticles that are less than $100 \mathrm{~nm}$ in diameter. All the photoluminescence results confirmed the presence of the lanthanide complexes in the final nanoparticles and demonstrated the potential of the employed chemical method to yield luminescent $\mathrm{SiO}_{2}$ nanoparticles. Moreover, the differences in the ${ }^{5} \mathrm{D}_{0}$ lifetimes and quantum efficiency and profile, energy, and fwhm of the ${ }^{5} \mathrm{D}_{0}$ $\rightarrow{ }^{7} \mathrm{~F}_{0-2}$ transitions between the nanoparticles and their starting complexes clearly indicate modifications in the $\mathrm{Eu}^{3+}$ local environment when the complexes were embedded in silica matrices. Clearly, this work showed that heteronuclear lanthanide complexes with adjusted visible photoluminescence could be encapsulated in nanosized $\mathrm{SiO}_{2}$. Thus, this method provides an alternative strategy to the synthesis of photoluminescent particles containing lanthanide species. ${ }^{30,31}$ In particular, the biofunctionalization of systems similar to 
those reported here might have interest for in vitro applications, such as optical biotagging.

Acknowledgment. We are grateful to R. A. S. Ferreira for help with the photoluminescence measurements, to $\mathrm{M}$. A. Martins for some of the SEM images and EDX maps, and to A. V. Girão for assistance on the TEM. This work was funded by Fundação para a Ciência e a Tecnologia (FCT) and FEDER (Project PTDC/QUI/67712/2006). P.C.R.S.-S. thanks FCT for the postdoctoral scholarship, SFRH/BPD/14954/2004. We also thank some of the TEM work to REDE/1509/RME/2005 and the team involved in this project.

Supporting Information Available: EDX maps of 3, 4, and 5 and additional excitation and emission spectra of $2 / \mathrm{SiO}_{2}-R$, $\mathbf{3}-\mathbf{5} / \mathrm{SiO}_{2}-R$, and $\mathbf{6}-\mathbf{8} / \mathrm{SiO}_{2}-10$. This material is available free of charge via the Internet at http://pubs.acs.org.

\section{References and Notes}

(1) Debuigne, F.; Cuisenaire, J.; Jeunieau, L.; Masereel, B.; Nagy, J. B. J. Colloid Interface Sci. 2001, 243, 90.

(2) Anker, J. N.; Behrend, C. J.; Huang, H. M.; Kopelman, R. J. Magn. Magn. Mater. 2005, 293, 655.

(3) Smith, A. M.; Dave, S.; Nie, S. M.; True, L.; Gao, X. H. Expert Rev. Mol. Diagn. 2006, 6, 231.

(4) Sharpe, M. J. Environ. Monit. 2003, 5, 109N. R107.

(5) Weiss, J.; Takhistov, P.; McClements, J. J. Food Sci. 2006, 71,

(6) Tan, W. H.; Wang, K. M.; He, X. X.; Zhao, X. J.; Drake, T.; Wang, L.; Bagwe, R. P. Med. Res. Rev. 2004, 24, 621.

(7) Burns, A.; Ow, H.; Wiesner, U. Chem. Soc. Rev. 2006, 35, 1028.

(8) You, C. C.; Verma, A.; Rotello, V. M. Soft Matter 2006, 2, 190.

(9) Darbandi, M.; Nann, T. Chem. Commun. 2006, 776.

(10) Kumar, A. R.; Hota, G.; Mehra, A.; Khilar, K. C. AIChE J. 2004, $50,1556$.
(11) Lal, M.; Chhabra, V.; Ayyub, P.; Maitra, A. J. Mater. Res. 1998, 13,1249

(12) Qhobosheane, M.; Santra, S.; Zhang, P.; Tan, W. H. Analyst 2001, $126,1274$.

(13) Voigt, A.; Adityawarman, D.; Sundmacher, K. Nanotechnology 2005,16, S429.

(14) Fioretto, D.; Freda, M.; Mannaioli, S.; Onori, G.; Santucci, A. J. Phys. Chem. B 1999, 103, 2631.

(15) Fu, X.; Qutubuddin, S. Colloids Surf., A 2001, 179, 65.

(16) Ng, W. B.; Wang, J.; Ng, S. C.; Gan, L. M. J. Am. Ceram. Soc. 1999, $82,529$.

(17) Meier, W. Curr. Opin. Colloid Interface Sci. 1999, 4, 6.

(18) Zhang, B.; Li, G. H.; Zhang, J.; Zhang, Y.; Zhang, L. D. Nanotechnology 2003, 14, 443.

(19) Darbandi, M.; Lu, W. G.; Fang, J. Y.; Nann, T. Langmuir 2006, 22, 4371 .

(20) Darbandi, M.; Thomann, R.; Nann, T. Chem. Mater. 2005, 17, 5720.

(21) Wang, F.; Tan, W. B.; Zhang, Y.; Fan, X. P.; Wang, M. Q. Nanotechnology 2006, 17, R1.

(22) Zhang, H.; Xu, Y.; Yang, W.; Li, Q. G. Chem. Mater. 2007, 19, 5875 .

(23) Dosev, D.; Nichkova, M.; Liu, M. Z.; Guo, B.; Liu, G. Y.; Hammock, B. D.; Kennedy, I. M. J. Biomed. Opt. 2005, 10, 64006.

(24) Hong, G. Y.; Yoo, K.; Moon, S. J.; Yoo, J. S. J. Electrochem. Soc. 2003, $150, \mathrm{H} 67$.

(25) Soares-Santos, P. C. R.; Nogueira, H. I. S.; Felix, V.; Drew, M. G. B.; Ferreira, R. A. S.; Carlos, L. D.; Trindade, T. Chem. Mater. 2003, $15,100$.

(26) Fernandes, J. A.; Ferreira, R. A. S.; Pillinger, M.; Carlos, L. D. Jepsen, J.; Hazell, A.; Ribeiro-Claro, P.; Goncalves, I. S. J. Lumin. 2005, $113,50$.

(27) Carlos, L. D.; Malta, O. L.; Albuquerque, R. Q. Chem. Phys. Lett. 2006, 416, 238. 21.

(28) Malta, O. L.; Batista, H. J.; Carlos, L. D. Chem. Phys. 2002, 282,

(29) Carlos, L. D.; Ferreira, R. A. S.; Bermudez, V. D.; Ribeiro, S. J. L. Adv. Mater. 2009, 21, 509.

(30) Yu, M.; Lin, J.; Fang, J. Chem. Mater. 2005, 17, 1783.

(31) Kong, D. Y.; Wang, Z. L.; Lin, C. K.; Quan, Z. W.; Li, Y. Y.; Li, C. X.; Lin, J. Nanotechnology 2007, 18, 75601.

JP811363G 